care. These are excellent chapters and will be useful to clinicians evaluating specific syndromes. The lack of color pictures in the dermatologic and oral manifestations sections (even in the online version) is a drawback. The final chapters on drug administration and medications are useful tabulations of drug interactions, dosing, and adverse events.
This is an excellent comprehensive source book for AIDS clinicians, although it should not be considered a rapid guide to treatment options. This is a text that will be useful for understanding the basis of our current drug therapy. In contrast, the chapters discussing specific disease processes or syndromes will be extremely useful for the busy clinician looking for a single source for these conditions.

\author{
David Rimland* \\ *Veterans Administration Medical Center, \\ Decatur, Georgia, USA \\ Address for correspondence: David Rimland, \\ Veterans Administration Medical Center, 1670 \\ Clairmont Rd, Decatur, GA 30033, USA; email: \\ david.rimland@va.gov
}

\title{
Bedtime at Nana and Pop's House
}

\section{Stan Shuman}

Requires a hug and a kiss

From the two year old

In Mickey Mouse pajamas,

Climbing on my lap,

Interrupting the crime-news on T.V.,

Smack! a kiss on the left ear,

Smack! a kiss on the right,

"Eye, eye," the imp insists,

(Thank goodness for eyeglasses)

"Nose, nose," comes the next command.

I panic (what to do?!)

This adorable, cute, bright, affectionate kid,

My own grandchild,

Heading for the lips, now!

(What a strange ritual

The young parents have invented)

All I can think of, are GERMS:

Giardia, Hemophilus, E. coli,

Strep, Staph, and Pneumo,

A host of enterorespiratory viruses

Multiplying on this adorable child's pink

Mucous membranes, fingertips,

His droplets and aerosols a sea of microbes.
I suddenly thrust him

At arm's length, crown him

With a kiss on the curls

Of the cranium, blow

A few more long-distance

Kisses as I hand him

To his mother

(Before any more infestation can occur).

I return to the gloomy T.V.,

Wondering what the incubation

Periods are for the most likely

Forms of gastroenteritis, hepatitis,

Pink eye, U.R.I. and

Bronchopneumonia.

How fortunate the non-medical

Parents and co-grandparents,

Who hug, hug; kiss, kiss

Without worry or care!

Stan Schuman is professor emeritus at the Medical University of South Carolina, Charleston, SC, USA, and founding editor of the Journal of Agromedicine (Haworth Medical Press, New York, 1974). 